OPEN ACCESS

Edited by:

Wei Hu,

Fudan University, China

Reviewed by:

Francisco Bolas Fernandez, Complutense University of Madrid,

Spain

Xin Ping Zhu,

Capital Medical University, China

*Correspondence:

Zhong Q. Wang

wangzq@zzu.edu.cn

Jing Cui

cuij@zzu.edu.cn

Specialty section:

This article was submitted to

Infectious Diseases,

a section of the journal

Frontiers in Microbiology

Received: 10 March 2017 Accepted: 16 May 2017

Published: 31 May 2017

Citation:

Wang ZQ, Liu RD, Sun GG, Song $Y$, Jiang $P$, Zhang $X$ and Cui $J$

(2017) Proteomic Analysis

of Trichinella spiralis Adult Worm

Excretory-Secretory Proteins Recognized by Sera of Patients with

Early Trichinellosis.

Front. Microbiol. 8:986.

doi: 10.3389/fmicb.2017.00986

\section{Proteomic Analysis of Trichinella spiralis Adult Worm Excretory-Secretory Proteins Recognized by Sera of Patients with Early Trichinellosis}

\author{
Zhong Q. Wang*, Ruo D. Liu, Ge G. Sun, Yan Y. Song, Peng Jiang, Xi Zhang and \\ Jing Cui*
}

Department of Parasitology, Medical College, Zhengzhou University, Zhengzhou, China

The most commonly used serodiagnostic antigens for trichinellosis are the excretorysecretory (ES) antigens from T. spiralis muscle larvae (ML), but the specific antibodies against the ML ES antigens are usually negative during early stage of Trichinella infection. The recent studies demonstrated that $T$. spiralis adult worm (AW) antigens were recognized by mouse or swine infection sera on Western blot as early as 715 days post-infection (dpi), the AW antigens might contain the early diagnostic markers for trichinellosis. The purpose of this study was to screen early diagnostic antigens in T. spiralis AW ES proteins recognized by sera of early patients with trichinellosis. T. spiralis AW were collected at $72 \mathrm{~h}$ post-infection (hpi), and their ES antigens were analyzed by SDS-PAGE and Western blot. Our results showed that 5 protein bands (55, 48-50, 45, 44, and $36 \mathrm{kDa})$ were recognized by sera of early patients with trichinellosis collected at $19 \mathrm{dpi}$, and were subjected to shotgun LC-MS/MS and bioinformatics analyses. A total of 185 proteins were identified from $T$. spiralis protein database, of which 116 (67.2\%) proteins had molecular weights of $30 \sim 60 \mathrm{kDa}$, and 125 (67.6\%) proteins with pl 4-7. Bioinformatic analyses showed that the identified proteins have a wide diversity of biological functions (binding of nucleotides, proteins, ions, carbohydrates, and lipids; hydrolase, transferase, and oxidoreductase, etc.). Several enzymes (e.g., adult-specific DNase II, serine protease and serine protease inhibitor) could be the invasion-related proteins and early diagnostic markers for trichinellosis. Moreover, recombinant T. spiralis serine protease (rTsSP-ZH68) was expressed in E. coli and its antigenicity was analyzed by Western blot with the early infection sera. The rTsSP-ZH68 was recognized by sera of infected mice at 8-10 dpi and sera of early patients with trichinellosis at 19 dpi. T. spiralis AW proteins identified in this study, especially serine protease, are the promising early diagnostic antigens and vaccine candidates for trichinellosis.

Keywords: Trichinella spiralis, trichinellosis, adult worms, excretory-secretory antigens, immunoproteomics, early diagnosis 


\section{INTRODUCTION}

Trichinella spiralis, an intracellular parasitic nematode, can cause a serious foodborne trichinellosis. Humans acquire trichinellosis by ingesting raw or undercooked meat (mainly pork) that contains the infective larvae of Trichinella (Murrell, 2013). Human trichinellosis has been reported in 55 countries of the world and is considered as a re-emerging disease (Pozio, 2007). From 1986 to 2009, there were 65,818 cases and 42 deaths reported from 41 countries (Murrell and Pozio, 2011). In China, 17 outbreaks of human trichinellosis consisting of 828 cases were reported from 2000 to 2003, and 15 outbreaks of trichinellosis consisting of 1387 cases and 4 deaths occurred during 20042009 (Wang et al., 2006; Cui et al., 2011). Trichinellosis can lead to death, particularly in elderly patients accompany with neurological or cardiovascular complications. However, it is difficult to diagnose the human trichinellosis on the basis of clinical manifestations of the patients. (Dupouy-Camet et al., 2002).

Trichinella spiralis muscle larvae (ML) excretory-secretory (ES) antigens, recommended by the International Commission on Trichinellosis (ICT), are the most commonly used serodiagnostic antigens for trichinellosis (Gamble et al., 2004), but the specific antibodies against the ML ES antigens are usually negative during early stage of Trichinella infection (Cui et al., 2015), possibly because the majority of ML ES antigens are the stage-specific and not recognized by specific antibodies produced during the intestinal phase (Liu J.Y. et al., 2016). Previous studies have shown that $100 \%$ detection of anti-Trichinella IgG is not possible for at least 1-3 months after primary Trichinella infection (Bruschi et al., 2001). There is an obvious window period of 3-4 weeks between Trichinella infection and specific antibody positivity.

After being ingested, T. spiralis ML develop to intestinal infective larvae which invade the host's small intestinal epithelium, and undergo four molting to develop to adult worms (AW) in $31 \mathrm{~h}$ post-infection (hpi). After mating, the female AW invade intestinal mucosa again and live there for 1020 days in mice and rats or 4-6 weeks in human (Campbell, 1983). During the intestinal stage of T. spiralis infection, the ES antigens produced by the AW result in early exposure to the host's immune system and elicit the production of specific anti-Trichinella antibodies. The ES proteins of intestinal AW of $T$. spiralis might contain the early diagnostic markers of trichinellosis (Wang et al., 2017). The immunoproteomics study showed that 64 proteins in AW crude extracts were recognized by sera from $T$. spiralis-infected pig and mice at 7 days postinfection (dpi), and the recombinant Ts14-3-3 had the significant potential as early diagnostic reagents or vaccine candidates (Yang et al., 2015). The recombinant protein from pre-adults $20 \mathrm{hpi}$ was recognized by swine infection sera on Western blot as early as 15-20 dpi (Zocevic et al., 2014). In our previous studies, anti-Trichinella IgG of infected mice at $8 \mathrm{dpi}$ was detected by ELISA using AW ES antigens, while anti-Trichinella IgG cannot be detected before $12 \mathrm{dpi}$; meanwhile the detection of anti-Trichinella $\operatorname{IgG}$ of patients with trichinellosis at $19 \mathrm{dpi}$ demonstrated that the sensitivity of AW ES antigens (100\%) were superior to ML ES antigens (75\%) (Sun et al., 2015). Additionally, one protein band with $33 \mathrm{kDa}$ in T. spiralis AW ES antigens was recognized by sera of infected mice at $8 \mathrm{dpi}$ and 10 proteins of T. spiralis were identified by mass spectrometry (Liu R.D. et al., 2016). However, T. spiralis AW ES antigens recognized by sera of patients with trichinellosis have not yet been explored and they may contain the key target antigens important for host's immune recognition and diagnostic markers for early detection of Trichinella infection in humans. Therefore, it is possible to screen and characterize the new early diagnostic antigens from T. spiralis AW.

In this study, T. spiralis AW ES antigens were separated by SDS-PAGE and recognized by early sera of patients with trichinellosis in Western blot, then the recognized bands were selected for protein identification by shotgun LC-MS/MS analyses in combination with bioinformatics. These data are expected to provide valuable information for early diagnostic antigens for trichinellosis.

\section{MATERIALS AND METHODS}

\section{Ethics Statement}

This study was carried out in accordance with the National Guidelines for Experimental Animal Welfare (MOST of People's Republic of China, 2006). The protocol was approved by The Life Science Ethics Committee of Zhengzhou University. All individuals give oral informed consent before the use of serum samples from healthy donors and patients with trichinellosis.

\section{Parasites and Animals}

The T. spiralis isolate (ISS534) used in the present study was obtained from domestic pigs in Nanyang city of Henan Province, China. The Trichinella isolate was kept by the serial passage in BALB/c mice every 6-8 months in our laboratory. Specific pathogen-free (SPF) female BALB/c mice and Wistar rats were purchased from the Henan Provincial Experimental Animal Centre (Zhengzhou, China).

\section{Collection of T. spiralis AW and Preparation of AW ES Antigens}

Trichinella spiralis ML were collected from the skeletal muscles of infected mice at 42 dpi with an artificial digestion method (Gamble et al., 2000; Li et al., 2010). After recovery, the ML were used to orally inoculate 30 rats at 8,000 ML per rat. The AW were collected from the upper two-thirds (duodenum and jejunum) of small intestine of experimentally infected rats at 72 hpi (Sun et al., 2015). The collected AW were washed five times in PBS with $100 \mathrm{U}$ penicillin $/ \mathrm{ml}$ and $100 \mu \mathrm{g}$ streptomycin $/ \mathrm{ml}$ and then cultured at $37^{\circ} \mathrm{C}$ in RPMI 1640 and $5 \% \mathrm{CO}_{2}$ for $18 \mathrm{~h}$. After incubation, The supernatant contained ES proteins was obtained by centrifugation at $4^{\circ} \mathrm{C}, 11,000 \mathrm{~g}$ for $20 \mathrm{~min}$, and concentrated using an Amicon Ultra-3 Centrifugal Filter Unit (molecular weight [MW] cut-off, $3 \mathrm{kDa}$; Millipore, United States) at $4^{\circ} \mathrm{C}$ and 5, $000 \mathrm{~g}$ for $1 \mathrm{~h}$ (Liu R.D. et al., 2016). The ES antigen concentration was determined using the Bradford method and stored at $-80^{\circ} \mathrm{C}$ before use. 


\section{Serum Samples}

Serum samples from patients with trichinellosis were collected from the patients during two outbreaks with trichinellosis, which occurred in the Yunnan province located in southwestern China in 2003 (10 serum samples collected at 19 dpi) and 2013 (3 serum samples collected at 35 dpi) (Wang et al., 2006; Wang C.Q. et al., 2013). These patients had a history of ingestion of raw or undercooked meat and presented with typical clinical manifestations of trichinellosis (fever related with periorbital or facial oedema, myalgia, and eosinophilia). All these patients had serum specific anti-Trichinella IgG antibody by ELISA with ML ES antigens (Cui et al., 2015; Sun et al., 2015). The optical density values at $490 \mathrm{~nm}$ of ten serum samples from patients with early trichinellosis at 19 dpi were $0.54,0.535,0.585,0.615$, $0.545,0.585,0.56,0.59,0.53$, and 0.575 , respectively; the cut-off value was 0.45 . Serum samples from presumably healthy persons, who tested negative for anti-Trichinella IgG, were also included in the study. All the serum samples were stored at $-80^{\circ} \mathrm{C}$ until used.

The mouse infection sera were collected from 10 experimentally infected mice at $42 \mathrm{dpi}$, each mouse was orally infected with $100 \mathrm{~T}$. spiralis ML. Sera from uninfected mice were collected as negative controls and sera collected from the mice infected with T. spiralis at $42 \mathrm{dpi}$ were used as positive controls.

\section{SDS-PAGE and Western Blot Analysis}

The AW ES antigens $(20 \mu \mathrm{g})$ were subjected to sodium dodecyl sulfat-polyacrylamide gel (SDS-PAGE) in 5\% stacking gels and $12 \%$ resolving gels at $80 \mathrm{~V}$ for $40 \mathrm{~min}$ and 120 $\mathrm{V}$ for $90 \mathrm{~min}$. After electrophoresis, one gel was stained in Coomassie brilliant blue R-250 staining solution (Sigma, United States) for $4 \mathrm{~h}$, and the other gel was used for the electrotransfer of proteins on nitrocellulose (NC) membranes (Millipore, United States) at $18 \mathrm{~V}$ for $35 \mathrm{~min}$ via a semi-dry transfer cell (Bio-Rad, United States) (Wang B. et al., 2013). Subsequently, the membranes were cut into strips, blocked with $5 \%$ skim milk in TBST (20 mM Tris- $\mathrm{HCl}, 150 \mathrm{mM} \mathrm{NaCl}, 0.05 \%$ Tween-20, $\mathrm{pH} 7.6)$ at $37^{\circ} \mathrm{C}$ for $1 \mathrm{~h}$, and incubated at $4^{\circ} \mathrm{C}$ overnight with sera from different patients with trichinellosis at a dilution of 1:100. After being washed in TBST, the strips were incubated with HRP-conjugated goat anti-human IgG (1:5,000 dilutions; Sigma, United States) at $37^{\circ} \mathrm{C}$ for $1 \mathrm{~h}$. Finally, the immunoreaction was detected with 3, 3-diaminobenzidine tetrahydrochloride (DAB; Sigma, United States). The gel and membranes were scanned using ImageScanner (GE healthcare, United States) and the MW of these bands was analyzed by AlphaView software (ProteinSimple, Santa Clara, CA, United States).

\section{Mass Spectrometry Analysis}

Protein bands recognized by sera of early patients with trichinellosis were excised from the parallel gel and subjected to in-gel tryptic digestion according to an optimized procedure (Wang et al., 2012). Briefly, the gel was washed two times, $10 \mathrm{~min}$ each, with Milli-Q water and then destained with $25 \mathrm{mM}$
$\mathrm{NH}_{4} \mathrm{HCO}_{3}$ in $50 \%$ acetonitrile at $37^{\circ} \mathrm{C}$ until depigmentation. Then, the dried gel was incubated with $10 \mathrm{mM}$ dithiothreitol at $56^{\circ} \mathrm{C}$ for $1 \mathrm{~h}$ and alkylated with $55 \mathrm{mM}$ iodoacetamide at room temperature for $45 \mathrm{~min}$ in the dark. Gel pieces were washed two times with $100 \mu \mathrm{L}$ of $25 \mathrm{mM} \mathrm{NH}_{4} \mathrm{HCO}_{3}$ for $10 \mathrm{~min}$, destained as above for $10 \mathrm{~min}$ and then dehydrated in a vacuum centrifuge. Subsequently, the gel pieces were digested in $20 \mathrm{ng} / \mu \mathrm{l}$ trypsin buffers at $37^{\circ} \mathrm{C}$ overnight, followed by dissolved in $0.1 \%$ formic acid for shotgun analysis. Then, the proteins were separated by high performance liquid chromatography and analyzed by tandem MS, as previously described (Liu et al., 2010). Peptides were ionized in positive ion mode and introduced into the Q-Exactive Orbitrap mass spectrometer (Thermo Fisher Scientific, Waltham, MA, United States). Initial separation was performed on a PepMap C18 reverse phase capillary column (3 $\mu \mathrm{m}$ Hypersil C18, $75 \mu \mathrm{m} \times 2 \mathrm{~cm}$, Thermo Fisher Scientific, Waltham, MA, United States). Mobile phases were $0.1 \%$ FA in Millipore water as buffer A and $0.1 \%$ FA in $84 \%$ ACN as buffer B. Peptides were eluted using a 65 min gradient of $5-80 \%$ buffer $B$ at a flow rate of $400 \mathrm{~nL} / \mathrm{min}$. The $\mathrm{MS} / \mathrm{MS}$ data were searched against the T. spiralis protein database from UniProt and NCBI using the SEQUEST algorithm. The SEQUEST search parameters used in this study were Delta $\mathrm{CN}(\geq 0.1)$ and Xcorr (one charge $\geq 1.9$, two charges $\geq 2.2$, and three charges $\geq 3.75$ ) (Liu et al., 2015).

\section{Bioinformatics Analysis}

The signal peptide and transmembrane domains of the identified proteins were predicted by using online tool SignalP ${ }^{1}, \mathrm{TMHMM}^{2}$. To assign a possible function, gene ontology (GO) features of the identified proteins were obtained using Uniprot and InterPro databases (Mitchell et al., 2015). Then, the Web Gene Ontology Annotation Plotting $\left(\mathrm{WEGO}^{3}\right)$ was used to analysis the GO categories (Ye et al., 2006).

\section{Expression and Immunological Test of Recombinant T. spiralis Serine Protease (rTsSP-ZH68)}

Trichinella spiralis TsSP-ZH68 gene was amplified by PCR using specific primers with BamHI and Pstl restriction enzyme sites (5'-TTCGGATCCAATTATGAATGTG GCACCTTAC-3' and $5^{\prime}$-CCGCTGCAGTTAACGGAAAAAAGTGAATGAT-3'). The amplified PCR products were purified, digested, and cloned into the pGEM-T vector (Promega, United States) and subsequently sub-cloned into the expression vector PQE-80L (New England Biolabs, United States). The recombinant plasmid PQE-80L/TsSP-ZH68 was transformed into the Escherichia coli BL21. The rTsSP-ZH68 was expressed and identified in our laboratory (unpublished data). The purified rTsSP-ZH68 was identified by SDS-PAGE and transferred onto the NC membrane for Western blot analysis. The membrane was incubated with sera of the infected mice or sera of patients with trichinellosis to identify the immunogenic antigens.

\footnotetext{
${ }^{1}$ http://www.cbs.dtu.dk/services/SignalP/

${ }^{2} \mathrm{http} / / /$ www.cbs.dtu.dk/services/TMHMM/

${ }^{3} \mathrm{http} / / /$ wego.genomics.org.cn/cgi-bin/wego/index.pl
} 
A

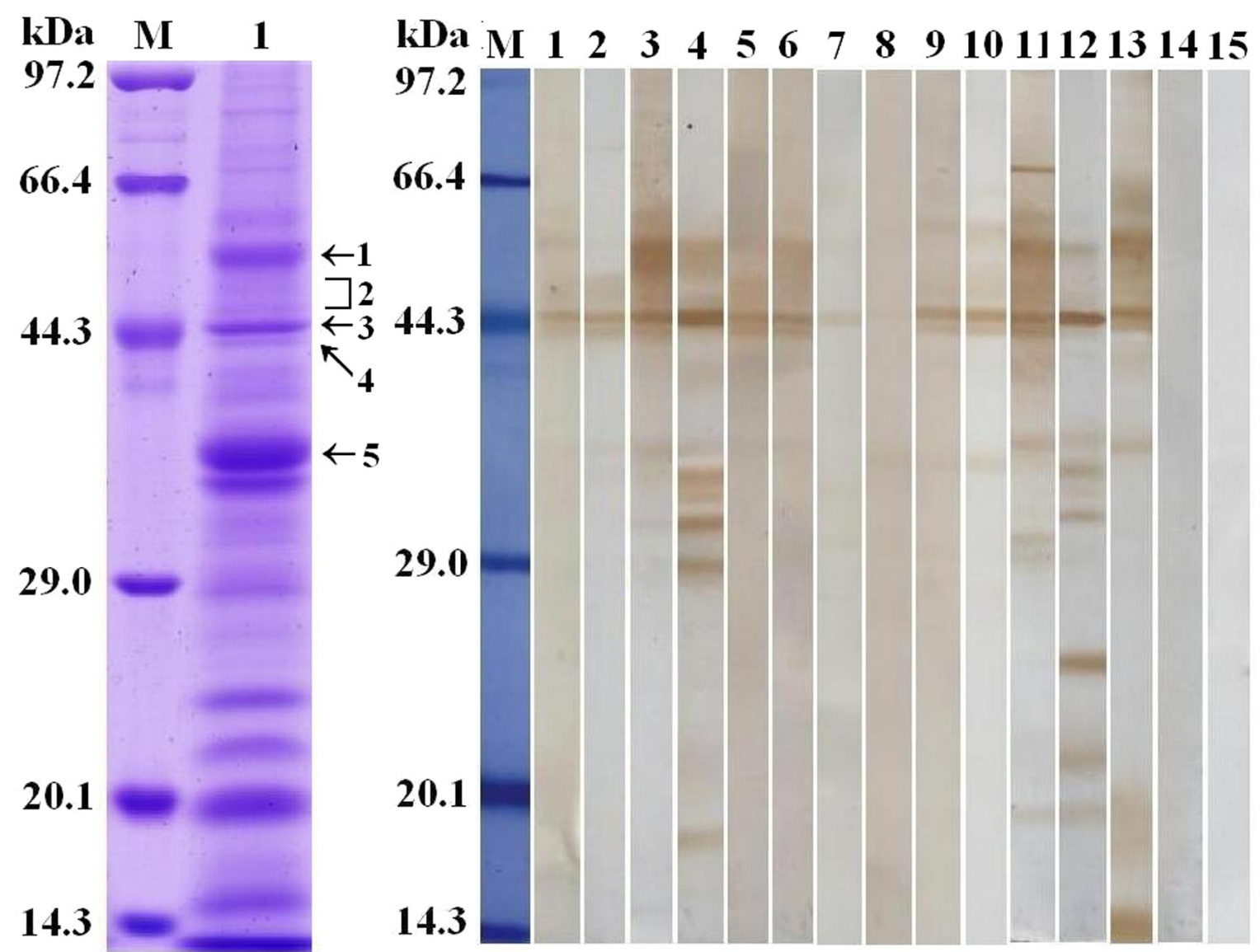

FIGURE 1 | Analysis of T. spiralis AW ES proteins recognized by sera of early patients with trichinellosis. (A) SDS-PAGE analysis of T. spiralis AW ES proteins. AW ES proteins were separated on $12 \%$ polyacrylamide gels. Lane $\mathrm{M}=$ low molecular weight protein marker; Lane $1=$ AW ES proteins. The arrows indicate the 5 protein bands (55, 48-50, 45, 44, and $36 \mathrm{kDa}$ ) which were excised, digested, and analyzed by LC-MS/MS. (B) Western blotting of AW ES proteins probed by sera of early patients with trichinellosis. Lane $\mathrm{M}=$ low molecular weight protein marker; Lane1-10 = anti-Trichinella lgG-positive sera from early patients with trichinellosis at 19 dpi; Lane 11-13 = anti-Trichinella IgG-positive sera from patients with trichinellosis at 35 dpi; Lane 14-15 = sera from healthy persons.

\section{RESULTS}

\section{SDS-PAGE and Western Blotting Analysis}

Trichinella spiralis AW ES proteins were separated by SDS-PAGE. The results demonstrated that the AW ES proteins had 25 bands with a $\mathrm{MW}$ ranging from 15 to $97 \mathrm{kDa}$ (Figure 1A). The results of Western blot analysis indicated that fifteen protein bands (77, $61,55,50,48,45,44,42,36,35,33,32,29,27$, and $17 \mathrm{kDa})$ were recognized by sera of early patients with trichinellosis at $19 \mathrm{dpi}$, and sixteen protein bands $(77,61,55,50,48,45,44,42$, $36,35,33,32,29,24,20$, and $17 \mathrm{kDa}$ ) were recognized by sera of patients with trichinellosis at $35 \mathrm{dpi}$ (Figure 1B). But, sera from healthy persons could not recognize these protein bands. The five protein bands $(55,48-50,45,44$, and $36 \mathrm{kDa})$ recognized by eight serum sample of early patients with trichinellosis were, respectively, used to the shotgun LC-MS/MS analysis.

\section{Protein Identification by Shotgun LC-MS/MS Analysis}

The result of shotgun LC-MS/MS analysis showed that total of $185 \mathrm{~T}$. spiralis proteins with unique pep counts $\geq 2$ were identified from T. spiralis protein database, of which 133 (71.89\%) proteins were annotated by InterProscan software. Several enzymes (e.g., adult-specific DNase II, serine protease and serine protease inhibitor) might be the invasion-related proteins and early diagnostic antigens for trichinellosis, and part of the proteins identified were shown in Table 1. The MW of 185 proteins varied from 7.38 to $493.65 \mathrm{kDa}$ with $116(62.70 \%)$ proteins distributed in the range of $30 \sim 60 \mathrm{kDa}$ (Figure 2). The $\mathrm{pI}$ value ranged from 3.98 to 9.90, with $80.54 \%$ of proteins distributed between 5 and 9. Out of 185 proteins, 48 (25.95\%) proteins had signal peptide and $37(20.00 \%)$ had a transmembrane domain (Supplementary Table 1). 


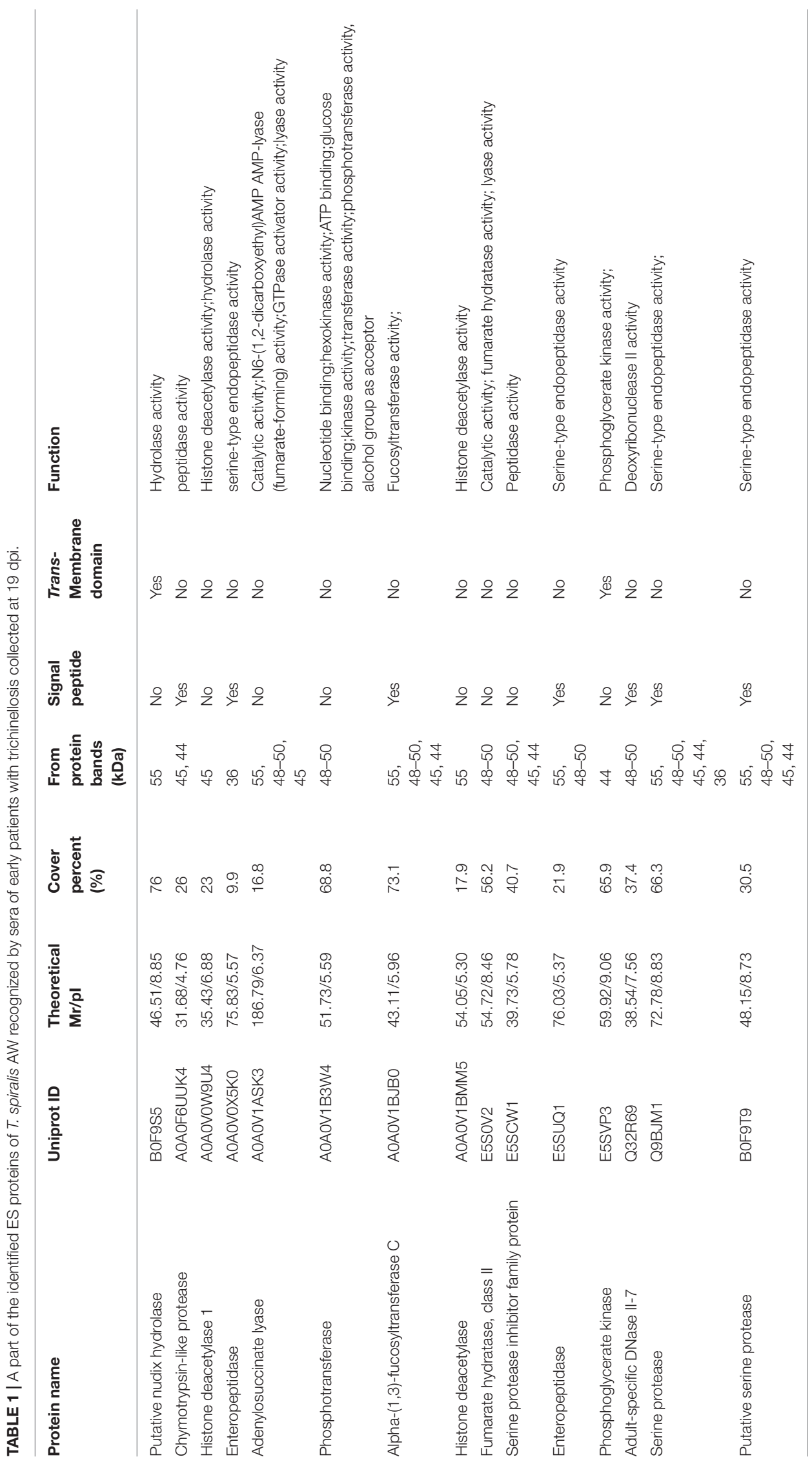



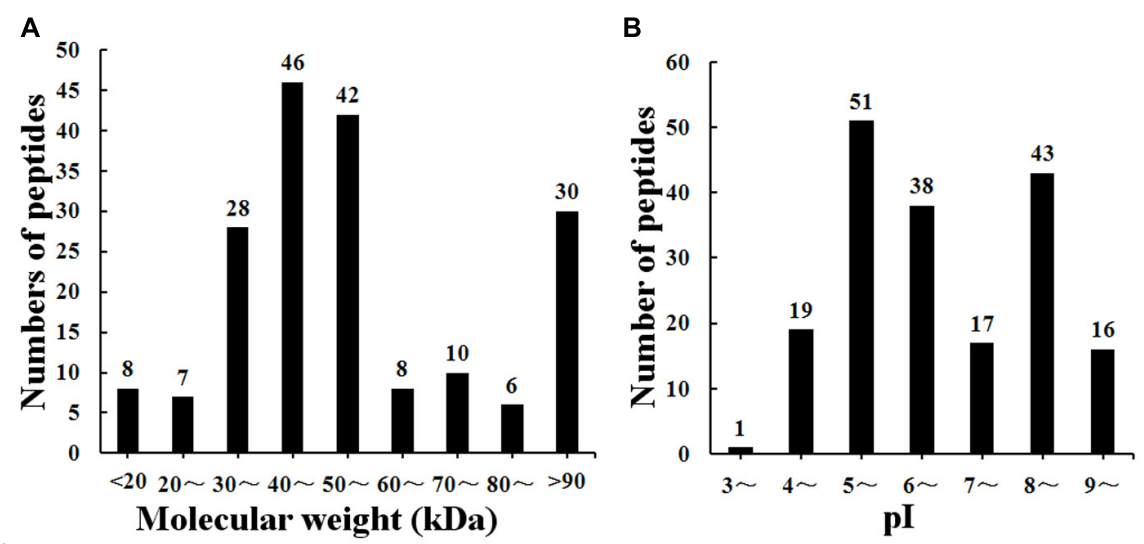

\section{C}

FIGURE 2 | Distribution of identified 185 T. spiralis proteins of AW ES proteins recognized by sera of early patients with trichinellosis collected at 19 dpi. (A) Molecular weight (MW). (B) Isoelectric point (pl). (C) Two-dimensional (MW and pl).

\section{Function Assignment in Identified Proteins through Gene Ontology}

To assign a possible function, the GO features of the 133 proteins with GO annotation were obtained by using Uniprot and InterProscan databases. The WEGO tool was applied to plot the GO annotation distribution (Figure 3). These proteins were grouped into three hierarchically structured GO terms, namely biological process, cellular component, and molecular function. The main biological process of these proteins was metabolic process (98 proteins, $73.68 \%$ ) and cellular process (79 proteins, 59.40\%). In the cellular component ontology, a large proportion of proteins were related to cell part (62 proteins, 46.62\%) and intracellular (44 proteins, 33.08\%). For molecular function category, catalytic activity (101 proteins, 75.94\%) and binding (58 proteins, 43.61\%) were the two main molecular function categories. The assigned catalytic activity of the 101 proteins could be assigned to hydrolase, transferase, oxidoreductase, isomerase, ligase, and lyase activity. This analysis indicated that the identified proteins involved in these GO categories might play the important roles in larval invasion of host intestinal epithelium cells (IECs) and immune escape.

\section{Recognition of rTsSP-ZH68 by Early Infection Sera}

To validate the potential of TsSP-ZH68 as early diagnostic antigen or vaccine target, the TsSP-ZH68 was successfully cloned and expressed in BL21. As shown in Figure 4A, the rTsSP-ZH68 was highly induced in E. coli BL21 in insoluble fractions. The MW (45.3 kDa) of the rTsSP-ZH68 (with the histidine tag) was identical with the predicted combined size of the protein encoded by TsSP-ZH68 gene and the histidine tag from the plasmid. Western blot analysis showed that the purified rTsSP-ZH68 was recognized by sera of infected mice at $8-10,15$, and $42 \mathrm{dpi}$, sera of early patients with trichinellosis at 19 and $35 \mathrm{dpi}$, and anti-His tag mouse monoclonal antibody, but not by sera from normal mice and healthy persons (Figure 4B).

\section{DISCUSSION}

In this study, an immunoproteomics approach was used to identify potential early diagnostic antigens or candidate vaccine target for trichinellosis. The AW ES proteins were analyzed by using SDS-PAGE and Western blotting, the five proteins bands $(55,48-50,45,44$, and $36 \mathrm{kDa})$ were recognized by sera of early patients with trichinellosis at $19 \mathrm{dpi}$. The results further demonstrated that the AW ES antigens might be secreted by the parasite into the host's peripheral blood circulation at early phase of Trichinella infection and induced an early specific antibody response (Sun et al., 2015; Liu R.D. et al., 2016). The five protein bands recognized by early infection sera were further characterized by shotgun LC-MS/MS. A total of 185 proteins of T. spiralis were identified, and several enzymes (e.g., adultspecific DNase II, serine protease and serine protease inhibitor) could be the invasion-related proteins and early diagnostic antigens for trichinellosis. Then, the identified proteins were further analyzed by GO annotation to provide a comprehensive understand. The identified proteins have a wide diversity of biological functions (binding of nucleotides, proteins, ions, carbohydrates and lipids; activity of hydrolase, transferase and oxidoreductase, lyase, etc.).

The subcategory of transferase activity included phosphotransferase, phosphoglycerate kinase, alpha- $(1,3)$ fucosyltransferase C, citrate synthase, etc. Fucosylated glycans are produced by fucosyltransferases (FTs), which are a group of enzymes responsible for the transfer of fucose from guanosme 5diphosphate-a $(1-3,4)$ fucosidase (GDP-Fuc) to oligosaccharide acceptors linked to protein and lipid. Fucosylated glycans play the major roles in the development, survival and adaptation of Schistosoma mansoni in its hosts (Trottein et al., 2000; Hokke and Yazdanbakhsh, 2005; Mickum et al., 2016). The recent studies also demonstrated that anti-schistosomal candidate molecules including fucosyltransferases via life cycle transcriptome analyses and gene microarray (Fitzpatrick et al., 2009; Protasio et al., 2012). The subcategory of lyase activity included adenylosuccinate lyase (ASL), diphosphomevalonate 


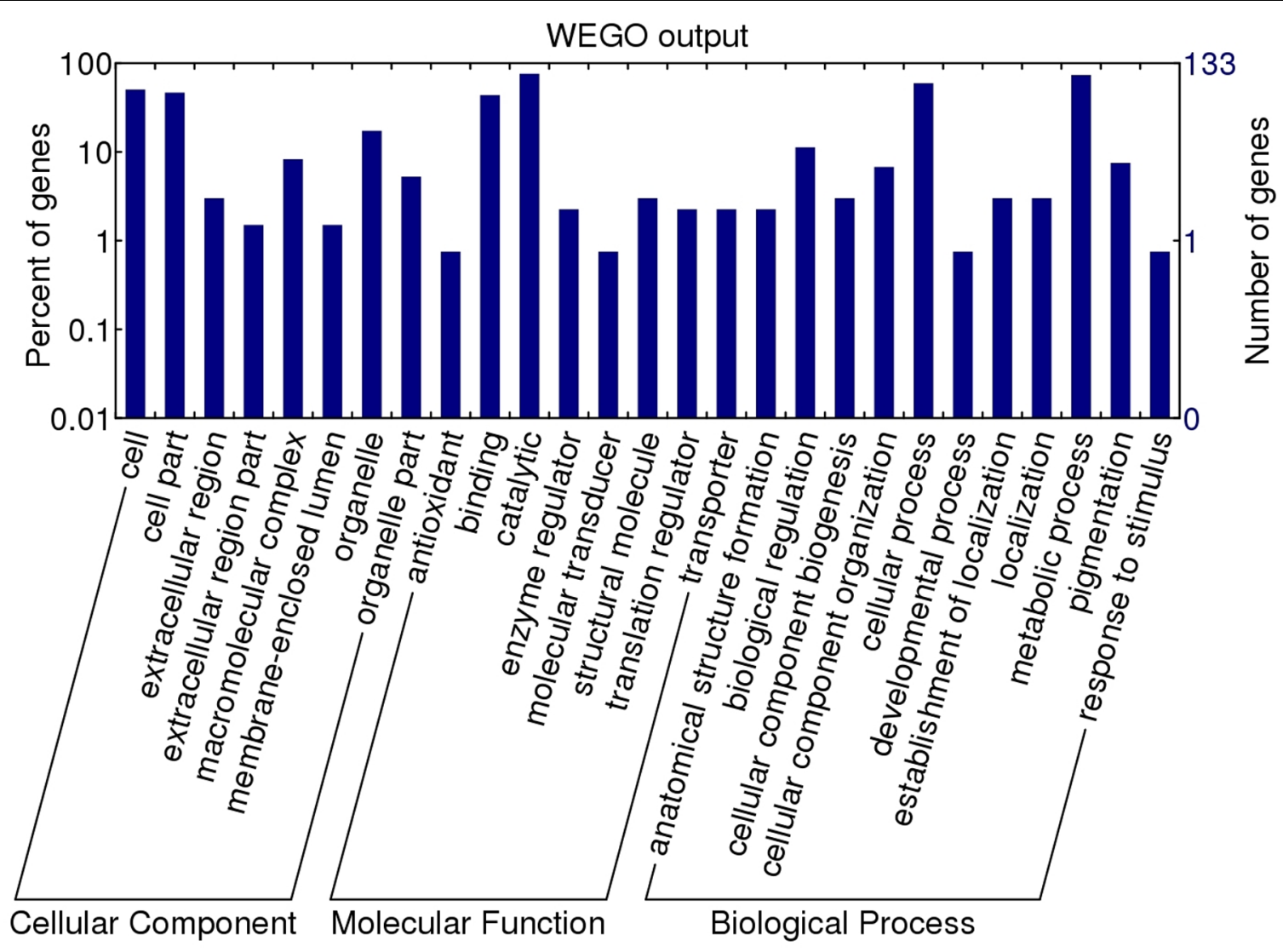

FIGURE 3 | Gene ontology categories of the identified T. spiralis AW ES proteins recognized by sera of early patients with trichinellosis collected at 19 dpi. The proteins were grouped into cellular component, molecular function, and biological process in accordance with their GO signatures. The right-hand showed the number of proteins with GO annotations. The percent of genes denotes that the proportion of GO terms in total genes.

A

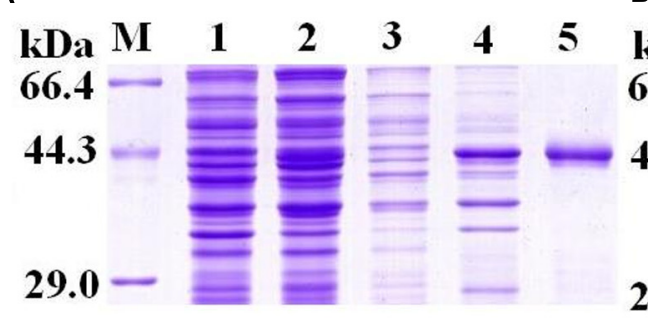

B

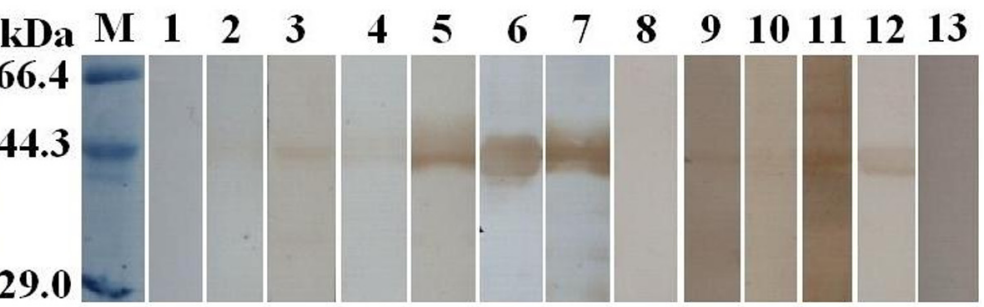

FIGURE 4 | Sodium dodecyl sulfat-polyacrylamide gel and Western blot analysis of rTsSP-ZH68 antigenicity. (A) SDS-PAGE analysis of rTsSP-ZH68. Lane M = low molecular weight protein marker; Lane 1 = non-induced recombinant bacteria lysate; Lane 2 = IPTG-induced recombinant bacteria lysate; Lane 3 = soluble fraction of induced recombinant bacteria; Lane 4 = insoluble fraction of induced recombinant bacteria; Lane $5=$ purified rTsSP-ZH68. (B) Western blot analysis of rTsSP-ZH68 antigenicity. Lane 1-4 = sera of mice infected with T. spiralis at 7-10 dpi; Lane $5=$ sera of mice infected with $T$. spiralis at 15 dpi; Lane $6=$ sera of mice infected with T. spiralis at 42 dpi; Lane 7 = anti-His tag mouse monoclonal antibody; Lane 8 = sera of normal mice; Lane 9-10 = anti-Trichinella lgG- positive sera from early patients with trichinellosis at 19 dpi; Lane 11-12 = anti-Trichinella IgG-positive sera from patients with trichinellosis at 35 dpi; Lane $13=$ sera from healthy persons.

decarboxylase and fumarate hydratase class II. ASL is an enzyme in parasite nucleotide salvage pathways that cleaves adenylosuccinate into adenosine 5'-monophosphate and fumarate (Senft et al., 1972; Foulk et al., 2002). In Leishmania donovani, ASL is central enzymes in purine salvage and has been validated as a potential drug target (Boitz et al., 2013). Schistosomes rely on purine salvage pathways to obtain nucleotides, so the enzymes of schistosome purine salvage pathways are the molecular targets for anti-schistosomal drugs (Senft et al., 1972; Senft and Crabtree, 1983). In S. mansoni, 
ASL is a crucial enzyme and should be evaluated as a possible chemotherapeutic target (Foulk et al., 2002). In this study, alpha-(1,3)-fucosyltransferase C and ASL was recognized by sera of early patients with trichinellosis, suggesting that they have potential to be targets for vaccine or immunodiagnostic antigens.

In the hydrolase activity subcategory, 60 proteins had hydrolase activity, including serine protease, cytosol aminopeptidase, chymotrypsin-like protease, chymotrypsinlike protease and enteropeptidase, of which the putative serine protease (TsSP-ZH68; uniprot ID: B0F9T9) is of particular interest. The TsSP-ZH68 belongs to peptidase S1A, chymotrypsin family, and chymotrypsin is a digestive enzyme of pancreatic juice in the duodenum where it carries out proteolysis, the breakdown of proteins and polypeptides (Wilcox, 1970). Serine proteases with chymotrypsin-like, elastase-like or trypsin-like activities are the most abundant protease of $T$. spiralis ES products or crude extract (Todorova and Stoyanov, 2000; Wang et al., 2014; Yang et al., 2015b; Liu J.Y. et al., 2016). Serine proteases play a variety of roles during the parasite life cycle, such as parasite development and nutrition, host tissue and cell invasion, anticoagulation, and immune evasion (Yang et al., 2015b). NBL1 is a newborn larva stage-specific serine protease and C-terminal part of NBL1 (NBL1-C) can be useful for the early diagnosis of trichinellosis in pigs, invasion of host cells and protective immune responses during Trichinella infection (Yang et al., 2015a, 2016). T. spiralis serine protease-1 named TspSP-1 contributes to the movement of the larvae by degrading cytoplasmic or intercellular proteins (Romaris et al., 2002). TspSP-1.2 could play an important role in the larval invasion of host's intestinal mucosa and might be a potential candidate target for vaccine against T. spiralis infection (Wang B. et al., 2013). The $31 \mathrm{kDa}$ proteins screened from T. spiralis ML ES proteins by immunoproteomics, belongs to the trypsin-like serine protease superfamily, possess significant potential as early diagnostic antigen for trichinellosis (Cui et al., 2015). The TsSP-ZH68 identified in this study was chosen for expression in recombinant protein in $E$. coli to further evaluate as a reagent for the early serodiagnosis for trichinellosis. The results demonstrated that TsSP-ZH68 is feasible to be expressed as insoluble recombinant protein in E. coli and the purified rTsSPZH68 from induced bacterial lysates was recognized by sera of infected mice at 8-10 dpi and sera of early patients with trichinellosis at $19 \mathrm{dpi}$. These results further indicated that TsSPZH68 is an immunodominant antigen recognized by sera of early patients with trichinellosis and could be a good diagnostic marker for early trichinellosis. The sensitivity and specificity of

\section{REFERENCES}

Boitz, J. M., Strasser, R., Yates, P. A., Jardim, A., and Ullman, B. (2013). Adenylosuccinate synthetase and adenylosuccinate lyase deficiencies trigger growth and infectivity deficits in Leishmania donovani. J. Biol. Chem. 288, 8977-8990. doi: 10.1074/jbc.M112.431486

Bruschi, F., Moretti, A., Wassom, D., and Fioretti, D. P. (2001). The use of a synthetic antigen for the serological diagnosis of human trichinellosis. Parasite 8, S141-S143. doi: 10.1051/parasite/200108s2141
rTsSP-ZH68 for detecting anti-Trichinella IgG need to be further investigated by using the sera of patients with trichinellosis, other helminthiases and healthy persons.

\section{CONCLUSION}

In this study, the ES proteins of T. spiralis AW were identified and characterized by immunoproteomic analysis with sera of early patients with trichinellosis. A total of 185 proteins in five protein bands $(55,48-50,45,44$ and $36 \mathrm{kDa})$ were identified and analyzed by shotgun LC-MS/MS combined with bioinformatics. The AW ES proteins provided a new source of early diagnostic antigens for trichinellosis. The identified proteins (adult-specific DNase II, serine protease and serine protease inhibitor) might be the invasion-related proteins and early diagnostic antigens. The TsSP-ZH68 identified in this study possess significant potential as early serodiagnostic antigen, but its sensitivity and specificity need to be further studied by using sera of patients with trichinellosis and other helminthiases.

\section{AUTHOR CONTRIBUTIONS}

ZW and JC conceived and designed this study. RL, GS, YS, PJ, and XZ performed the experiments. ZW, RL, and JC wrote and revised the manuscript. All authors approved the final manuscript to be published.

\section{FUNDING}

This work was supported by the National Natural Science Foundation of China (no. 81572024 and 81672043).

\section{ACKNOWLEDGMENT}

The authors wish to thank Beijing Protein Innovation Co., Ltd. for the technology support.

\section{SUPPLEMENTARY MATERIAL}

The Supplementary Material for this article can be found online at: http://journal.frontiersin.org/article/10.3389/fmicb. 2017.00986/full\#supplementary-material

Campbell, W. C. (1983). Trichinella and Trichinosis. New York, NY: Plenum Press, 75-151. doi: 10.1007/978-1-4613-3578-8

Cui, J., Wang, L., Sun, G. G., Liu, L. N., Zhang, S. B., Liu, R. D., et al. (2015). Characterization of a Trichinella spiralis $31 \mathrm{kDa}$ protein and its potential application for the serodiagnosis of trichinellosis. Acta Trop. 142, 57-63. doi: 10.1016/j.actatropica.2014.10.017

Cui, J., Wang, Z. Q., and Xu, B. L. (2011). The epidemiology of human trichinellosis in China during 2004-2009. Acta Trop. 118, 1-5. doi: 10.1016/j.actatropica. 2011.02.005 
Dupouy-Camet, J., Kociecka, W., Bruschi, F., Bolas-Fernandez, F., and Pozio, E. (2002). Opinion on the diagnosis and treatment of human trichinellosis. Expert Opin. Pharmacother. 3, 1117-1130. doi: 10.1517/14656566.3.8.1117

Fitzpatrick, J. M., Peak, E., Perally, S., Chalmers, I. W., Barrett, J., Yoshino, T. P., et al. (2009). Anti-schistosomal intervention targets identified by lifecycle transcriptomic analyses. PLoS Negl. Trop. Dis. 3:e543. doi: 10.1371/journal. pntd.0000543

Foulk, B. W., Pappas, G., Hirai, Y., Hirai, H., and Williams, D. L. (2002). Adenylosuccinate lyase of Schistosoma mansoni: gene structure, mRNA expression, and analysis of the predicted peptide structure of a potential chemotherapeutic target. Int. J. Parasitol. 32, 1487-1495. doi: 10.1016/S00207519(02)00161-3

Gamble, H. R., Bessonov, A. S., Cuperlovic, K., Gajadhar, A. A., van Knapen, F., Noeckler, K., et al. (2000). International commission on trichinellosis: recommendations on methods for the control of Trichinella in domestic and wild animals intended for human consumption. Vet. Parasitol. 93, 393-408. doi: 10.1016/S0304-4017(00)00354-X

Gamble, H. R., Pozio, E., Bruschi, F., Nockler, K., Kapel, C. M. O., and Gajadhar, A. A. (2004). International commission on trichinellosis: recommendations on the use of serological tests for the detection of Trichinella infection in animals and man. Parasite 11, 3-13. doi: 10.1051/parasite/20041113

Hokke, C. H., and Yazdanbakhsh, M. (2005). Schistosome glycans and innate immunity. Parasite Immunol. 27, 257-264. doi: 10.1111/j.1365-3024.2005. 00781.x

Li, F., Cui, J., Wang, Z. Q., and Jiang, P. (2010). Sensitivity and optimization of artificial digestion in the inspection of meat for Trichinella spiralis. Foodborne Pathog. Dis. 7, 879-885. doi: 10.1089/fpd.2009.0445

Liu, J. S., Zheng, S. C., Liu, L., Li, L., and Feng, Q. L. (2010). Protein profiles of the midgut of Spodoptera litura larvae at the sixth instar feeding stage by shotgun ESI-MS approach. J. Proteome Res. 9, 2117-2147. doi: 10.1021/pr900826f

Liu, J. Y., Zhang, N. Z., Li, W. H., Li, L., Yan, H. B., Qu, Z. G., et al. (2016). Proteomic analysis of differentially expressed proteins in the three developmental stages of Trichinella spiralis. Vet. Parasitol. 231, 32-38. doi: 10.1016/j.vetpar.2016.06.021

Liu, R. D., Cui, J., Liu, X. L., Jiang, P., Sun, G. G., Zhang, X., et al. (2015). Comparative proteomic analysis of surface proteins of Trichinella spiralis muscle larvae and intestinal infective larvae. Acta Trop. 150, 79-86. doi: 10. 1016/j.actatropica.2015.07.002

Liu, R. D., Qi, X., Sun, G. G., Jiang, P., Zhang, X., Wang, L. A., et al. (2016). Proteomic analysis of Trichinella spiralis adult worm excretorysecretory proteins recognized by early infection sera. Vet. Parasitol. 231, 43-46. doi: 10.1016/j.vetpar.2016.10.008

Mickum, M. L., Rojsajjakul, T., Yu, Y., and Cummings, R. D. (2016). Schistosoma mansoni alpha 1,3-fucosyltransferase-F generates the Lewis $\mathrm{X}$ antigen. Glycobiology 26, 270-285. doi: 10.1093/glycob/cwv103

Mitchell, A., Chang, H. Y., Daugherty, L., Fraser, M., Hunter, S., Lopez, R., et al. (2015). The InterPro protein families database: the classification resource after 15 years. Nucleic Acids Res. 43, D213-D221. doi: 10.1093/nar/gku1243

Murrell, K. D. (2013). Zoonotic foodborne parasites and their surveillance. Rev. Sci. Tech. 32, 559-569. doi: 10.20506/rst.32.2.2239

Murrell, K. D., and Pozio, E. (2011). Worldwide occurrence and impact of human trichinellosis 1986-2009. Emerg. Infect. Dis. 17, 2194-2202. doi: 10.3201/ eid1712.110896

Pozio, E. (2007). World distribution of Trichinella spp. infections in animals and humans. Vet. Parasitol. 149, 3-21. doi: 10.1016/j.vetpar.2007.07.002

Protasio, A. V., Tsai, I. J., Babbage, A., Nichol, S., Hunt, M., Aslett, M. A., et al. (2012). A systematically improved high quality genome and transcriptome of the human blood fluke Schistosoma mansoni. PLoS Negl. Trop. Dis. 6:e1455. doi: 10.1371/journal.pntd.0001455

Romaris, F., North, S. J., Gagliardo, L. F., Butcher, B. A., Ghosh, K., Beiting, D. P., et al. (2002). A putative serine protease among the excretory-secretory glycoproteins of L1 Trichinella spiralis. Mol. Biochem. Parasitol. 122, 149-160. doi: 10.1016/S0166-6851(02)00094-4

Senft, A. W., and Crabtree, G. W. (1983). Purine metabolism in the schistosomes: potential targets for chemotherapy. Pharmacol. Ther. 20, 341-356. doi: 10.1016/ 0163-7258(83)90031-1

Senft, A. W., Miech, R. P., Brown, P. R., and Senft, D. G. (1972). Purine metabolism in Schistosoma mansoni. Int. J. Parasitol. 2, 249-260. doi: 10.1016/ 0020-7519(72)90013-6
Sun, G. G., Wang, Z. Q., Liu, C. Y., Jiang, P., Liu, R. D., Wen, H., et al. (2015). Early serodiagnosis of trichinellosis by ELISA using excretory-secretory antigens of Trichinella spiralis adult worms. Parasit. Vectors 8, 484. doi: 10.1186/s13071015-1094-9

Todorova, V. K., and Stoyanov, D. I. (2000). Partial characterization of serine proteinases secreted by adult Trichinella spiralis. Parasitol. Res. 86, 684-687. doi: 10.1007/PL00008552

Trottein, F., Mollicone, R., Fontaine, J., de Mendonça, R., Piller, F., Pierce, R., et al. (2000). Molecular cloning of a putative alpha3-fucosyltransferase from Schistosoma mansoni. Mol. Biochem. Parasitol. 107, 279-287. doi: 10.1016/ S0166-6851(00)00213-9

Wang, B., Wang, Z. Q., Jin, J., Ren, H. J., Liu, L. N., and Cui, J. (2013). Cloning, expression and characterization of a Trichinella spiralis serine protease gene encoding a $35.5 \mathrm{kDa}$ protein. Exp. Parasitol. 134, 148-154. doi: 10.1016/j. exppara.2013.03.004

Wang, C. Q., Wu, F. W., Wang, X. R., Xu, H. B., Li, E., and Long, G. P. (2013). Survey of an outbreak of trichinellosis in Lanchang county of Yunnan province. China Trop. Med. 13, 1433-1444.

Wang, L., Cui, J., Hu, D. D., Liu, R. D., and Wang, Z. Q. (2014). Identification of early diagnostic antigens from major excretory-secretory proteins of Trichinella spiralis muscle larvae using immunoproteomics. Parasit. Vectors 7:40. doi: 10. 1186/1756-3305-7-40

Wang, Z. Q., Cui, J., and Xu, B. L. (2006). The epidemiology of human trichinellosis in China during 2000-2003. Acta Trop. 97, 247-251. doi: 10.1016/j.actatropica. 2005.03.012

Wang, Z. Q., Shi, Y. L., Liu, R. D., Jiang, P., Guan, Y. Y., Chen, Y. D., et al. (2017). New insights on serodiagnosis of trichinellosis during window period: early diagnostic antigens from Trichinella spiralis intestinal worms. Infect. Dis. Poverty 6, 41. doi: 10.1186/s40249-017-0252-z

Wang, Z. Q., Wang, L., and Cui, J. (2012). Proteomic analysis of Trichinella spiralis proteins in intestinal epithelial cells after culture with their larvae by shotgun LC-MS/MS approach. J. Proteomics 75, 2375-2383. doi: 10.1016/j.jprot.2012. 02.005

Wilcox, P. E. (1970). Chymotrypsinogens-chymotrypsins. Methods Enzymol. 19, 64-108. doi: 10.1016/0076-6879(70)19007-0

Yang, J., Pan, W., Sun, X. M., Zhao, X., Yuan, G., Sun, Q., et al. (2015). Immunoproteomic profile of Trichinella spiralis adult worm proteins recognized by early infection sera. Parasit. Vectors 8, 20. doi: 10.1186/s13071015-0641-8

Yang, Y., Lacour, S. A., Laine-Prade, V., Versille, N., Grasset-Chevillot, A., Feng, S., et al. (2015a). Trichinella spiralis newborn larvae: characterization of a stage specific serine proteinase expression, NBL1, using monoclonal antibodies. Parasitology 142, 783-790. doi: 10.1017/S0031182014001851

Yang, Y., Vallee, I., Lacour, S. A., Boireau, P., Cheng, S. P., and Liu, M. Y. (2016). Identification and characterization of immunodominant linear epitopes on the antigenic region of a serine protease in newborn Trichinella larvae. J. Helminthol. 90, 232-237. doi: 10.1017/S0022149X15 000267

Yang, Y., Wen, Y. J., Cai, Y. N., Vallee, I., Boireau, P., Liu, M. Y., et al. (2015b). Serine proteases of parasitic helminths. Korean J. Parasitol. 53, 1-11. doi: 10. 3347/kjp.2015.53.1.1

Ye, J., Fang, L., Zheng, H. K., Zhang, Y., Chen, J., Zhang, Z. J., et al. (2006). WEGO: a web tool for plotting GO annotations. Nucleic Acids Res. 34, W293-W297. doi: 10.1093/nar/gkl031

Zocevic, A., Lacour, S. A., Mace, P., Giovani, B., Grasset-Chevillot, A., Vallee, I., et al. (2014). Primary characterization and assessment of a T. spiralis antigen for the detection of Trichinella infection in pigs. Vet. Parasitol. 205, 558-567. doi: 10.1016/j.vetpar.2014.09.004

Conflict of Interest Statement: The authors declare that the research was conducted in the absence of any commercial or financial relationships that could be construed as a potential conflict of interest..

Copyright (C) 2017 Wang, Liu, Sun, Song, Jiang, Zhang and Cui. This is an open-access article distributed under the terms of the Creative Commons Attribution License (CC BY). The use, distribution or reproduction in other forums is permitted, provided the original author(s) or licensor are credited and that the original publication in this journal is cited, in accordance with accepted academic practice. No use, distribution or reproduction is permitted which does not comply with these terms. 